\title{
Structure du verbe anglais : tableau explicatif
}

Alain Diana

\section{OpenEdition}

\section{Journals}

Édition électronique

URL : http://journals.openedition.org/asp/4252

DOI : 10.4000/asp.4252

ISSN : 2108-6354

\section{Éditeur}

Groupe d'étude et de recherche en anglais de spécialité

\section{Édition imprimée}

Date de publication : 1 octobre 1993

Pagination : $1-9$

ISSN : 1246-8185

\section{Référence électronique}

Alain Diana, «Structure du verbe anglais : tableau explicatif », ASp [En ligne], 2 | 1993, mis en ligne le 22 février 2014, consulté le 23 avril 2019. URL : http://journals.openedition.org/asp/4252 ; DOI : 10.4000/ asp.4252

Ce document a été généré automatiquement le 23 avril 2019.

Tous droits réservés 


\title{
Structure du verbe anglais : tableau explicatif
}

\author{
Alain Diana
}

1 Enseigner l'anglais à des étudiants d'électronique à l'I.U.T. amène certaines contraintes sur les objectifs en fonction du temps disponible et du niveau :

- une contrainte temporelle : comment présenter de manière simple des connaissances « élémentaires » comme certains aspects de la grammaire anglaise sachant que l'on dispose de cinquante heures d'anglais environ sur une année.

- une contrainte d'ordre psychologique : comment remédier à des lacunes souvent anciennes et bien ancrées que des révisions successives n'ont pas éradiquées?

2 Par exemple, comment présenter l'ensemble du verbe anglais et de ses auxiliaires de la manière la plus cohérente qui soit (en particulier pour une remise à niveau des groupes « faibles » ou « faux débutants » avec sept ou huit ans d'anglais). La forme de tableau m'a semblé la plus adaptée pour ces étudiants qui sont familiers de ce type de présentation et même demandeurs.

3 Les tableaux ci-dessous visent à leur offrir une description systématique de la structure du verbe en anglais, et à leur permettre dans un premier temps de corriger leurs erreurs de construction (du genre: * will can go ou *have making). Les tableaux seront donnés ici à la forme affirmative mais permettent par ailleurs de présenter l'interrogation et la négation. Ils peuvent éventuellement servir à améliorer la traduction des structures verbales dans le sens anglais/français, ce qui est important pour de futurs techniciens ayant à maîtriser une documentation technique (notices, etc.) souvent rédigée uniquement en anglais. 


\section{Études précédentes}

4 Il existe peu d'études sous forme de tableau clair et simple, aucune à ma connaissance qui reprenne l'ensemble des formes verbales d'une manière à la fois cohérente et accessible à tous. Je me contenterai de passer en revue trois d'entre elles.

5 B. Strang (1969: 161-167) propose une analyse en termes de variables indépendantes en opposition binaire: «These complicated distinctions... are best presented analytically in terms of a small number of binary oppositions of meaning» (p. 161). Elle donne les catégories suivantes :

1) non-interrogative (affirmative)/interrogative, 2) non-negative (positive) / negative, 3) non-passive (active)/passive, 4) non-durative/durative, 5) nonperfective/perfective. (1969: 163)

Elle y ajoute non-past/past, non-modal/modal (1969: 164-166) et même non-emphatic/ emphatic, non-mutative/mutative, etc. Cette présentation ne parait pas aussi nette que lorsqu'on utilise des colonnes. D'autre part, comme les étudiants ont souvent tendance à confondre $\mathbf{B E}+$ ing et $\mathbf{B E}+$ p.passé, une présentation séparée mais reprenant le même type de tableau me paraît plus efficace.

7 Hornby de son côté, après avoir noté qu'il ne saurait y avoir de définition adéquate du terme «verbe ", fait la distinction entre "finite and non-finite forms of verbs" $(1975: 1)$, sans autre justification que le fait qu'il est utile de les distinguer. Quant aux auxiliaires, " the term AUXILIARY is used for a number of verbs which have a variety of functions. » Pour le reste, «compound tenses... are made by combining two or more verb forms. » (voir p. 80-81 la conjugaison de « write » en douze catégories, divisées en « progressive » et «non-progressive »).

8 Quant à McCawley (1988), il explique de manière exhaustive ce qu'il entend par auxiliaire et verbe et en quoi il diffère de la position de Chomsky, mais son ouvrage ne s'adresse pas à des étudiants non spécialistes d'anglais langue seconde et $a$ fortiori encore moins à des « faux-débutants ».

\section{Formule de départ}

Chomsky (1957 : 44) donne la structure de l'auxiliaire sous la forme :

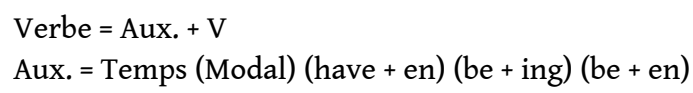

10 Cette formule a été largement reprise (Rivière 1988: 77). Mon idée au départ était simplement de la développer pour pouvoir visualiser toutes les possibilités. (On peut noter que ce type de présentation existe depuis plus de 20 ans pour l'allemand, avec le «cube » des formes verbales dans le Manuel du Germaniste). McCawley (1988) donne les conditions d'emploi de cette formule car certaines restrictions limitent les séquences possibles. Pourquoi donc réutiliser cette formule?

Pour des raisons " psychologiques"

11 Toute tentative d'approche «scientifique » des difficultés grammaticales est bien perçue par ce type d'étudiants, qui sont sensibles au fait que cela ressemble à une démonstration de type mathématique. Il n'y a donc pas de raison qu'ils ne « suivent » pas, dans la mesure où l'abstraction leur est familière. 
Pour des raisons pratiques

Parce qu'il ne s'agit pas d'en rester à une formulation abstraite, mais bien de la développer, et de "dérouler" toutes les formes actives et passives d'un verbe (sans toutefois tenir compte de l'acceptabilité des formes ainsi obtenues, en particulier au passif).

\section{Exploitation du tableau}

13 Il y a plusieurs manières d'aborder cette question globalement, et de préparer les étudiants. On peut, par exemple, leur demander de mettre par écrit toutes les formes d'un verbe simple qu'ils connaissent, puis vérifier leur exactitude, leur faire corriger les erreurs volontairement introduites dans une liste de formes verbales (ce qui est une bonne occasion de vérifier la sûreté de leurs connaissances).

Ensuite, on peut présenter le tableau comme un « menu déroulant » (par analogie avec les ordinateurs MacIntosh).

Pour des raisons pédagogiques, il m'a semblé plus judicieux de présenter séparément un tableau pour l'Actif et un pour le Passif. Si le niveau des étudiants est vraiment faible, on commence par leur présenter la « formule »: Aux. = Temps (Modal) (have + en) (be + ing), et par tracer 4 colonnes ${ }^{1}$. Puis on choisit un verbe régulier de préférence (ici : PLAY). Comme on laisse les 3 premières colonnes vides, on peut déjà demander aux étudiants comment la marque du temps va être signalée: ils trouvent assez facilement la distinction Présent/Passé.

Tableau 1

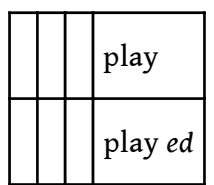

Tableau 2

Ensuite, on essaie de leur faire trouver la colonne à gauche, et on leur fait remarquer deux choses : a) si on choisit la colonne «BE », on trouve obligatoirement ING accolé au verbe suivant (à l'Actif) ; b) BE porte la marque Présent ou Passé uniquement.

Tableau3

\begin{tabular}{|l|l|l|}
\hline & ARE & play ING \\
\hline & WERE & play ING \\
\hline
\end{tabular}


Tableau 4

\section{De même avec HAVE : seule la forme la plus à gauche (ici Have) porte la marque présent/ passé. De plus, la colonne qui suit HAVE est obligatoirement au participe passé. Enfin, ne pas oublier qu'en 2 et en 4, BE exige ING derrière le verbe.}

\section{Tableau 5}

\begin{tabular}{|l|l|l|l|}
\hline HAVE & & play ED \\
\hline HAVE & BE EN & play ING \\
\hline HAD & & play ED \\
\hline HAD & BE EN & play ING \\
\hline
\end{tabular}

18 On peut inciter les étudiants plus avancés à trouver par eux-mêmes la suite du tableau. Avec les autres, on vérifiera si WILL est bien suivi de l'infinitif dans tous les cas, HAVE du participe passé et BE d'ING.

Tableau 6

\begin{tabular}{|l|l|l|l|}
\hline WILL & & & play \\
\hline WILL & & BE & play ING \\
\hline WILL & HAVE & & play ED \\
\hline WILL & HAVE & BE EN & play ING \\
\hline
\end{tabular}

19 Les explications données ici en commentaire du tableau semblent aller de soi, mais en réalité elles se révèlent absolument nécessaires pour des étudiants ayant des bases très fragiles. La présentation du tableau du Passif n'intervient que lorsque celui de l'Actif est parfaitement maîtrisé.

20 En guise de vérification, on peut faire refaire le même tableau pour d'autres verbes (avec des verbes irréguliers en particulier); donner une liste de formes verbales en mélangeant justes et fausses, et faire corriger les erreurs.

21 Il ne reste plus qu'à traduire le tableau en insérant chaque forme dans une phrase simple. Pour aider les étudiants très faibles, on leur demande de traduire de façon identique les formes avec et sans ING, simplification parfois abusive, mais qui leur permet d'appréhender plus rapidement les formes complexes.

22 D'un point de vue pratique, il serait souhaitable d'utiliser des couleurs différentes lors de la présentation pour faire ressortir « MODAUX + inf. », « HAVE + p.p. » et «BE + ing » (ce qui n'a pas été possible ici), car cela permet une visualisation plus efficace. L'utilisation de caractères différents aide malgré tout à bien les distinguer. 


\section{Limites et objections}

Des choix théoriques discutables?

$$
\begin{aligned}
& \text { always just HAVING RETURNED from a vacation ", que la formule de Chomsky ne prend pas } \\
& \text { en compte. Sauf pour des étudiants de niveau avancé, ces exemples restent très } \\
& \text { marginaux et ne nous concernent donc pas ici. }
\end{aligned}
$$
reproché à la grammaire générative. Mais après tout, nos étudiants n'ont-ils pas d'abord besoin de mécanismes simples?

Les passifs avec BE + BEing ou BEen + BEing sont très peu employés, et même tout à fait inutiles à ce niveau. Voir l'exemple donné par McCawley $(1988: 240):$ « Sam may have been being interrogated by the FBI ».

Qu'appelle-t-on auxiliaires ? Il est nécessaire de définir simplement ce que sont un verbe, un nom ou un adjectif pour certains étudiants, et il est également indispensable de définir ce qu'est un auxiliaire en respectant des critères simples (voir McCawley 1988, par exemple), car au-delà d'un niveau minimum, la question de la terminologie peut se révéler improductive ${ }^{3}$.

\section{Conclusion}

Cette manière de présenter la structure des verbes anglais a reçu un accueil positif de la plupart des étudiants auxquels ce tableau a été présenté à l'IUT. Les réactions sont plus difficiles à cerner pour des groupes de niveaux plus avancés, mais une bonne partie d'entre eux semble y avoir trouvé son compte, car ils ont eux aussi besoin de références précises.

31 Reste à savoir quelle est son efficacité réelle: au niveau de l'écrit, on constate une sensible diminution des erreurs portant sur la structure du groupe verbal. Pour l'instant, il n'est pas possible de dire si ce progrès est durable : il serait nécessaire de faire une enquête portant sur des groupes d'un niveau sensiblement égal utilisant ou non ce type de présentation et comparer les résultats obtenus sur une plus longue période de temps (six mois ou un an au moins). 


\section{BIBLIOGRAPHIE}

Beedham, C. 1987. « The English passive as an aspect ». Word 38/1, 7-12.

Bresson, D. 1989. « Langue, linguistique et grammaire. Mauvaises querelles et vrais problèmes ». Les Langues Modernes 3-4 « Théories linguistiques et pratiques grammaticales », 21-28.

Chomsky, N. 1957. Syntactic Structures. La Haye : Mouton.

Gauthier, A. 1981. Opérations énonciatives et apprentissage d'une langue étrangère en milieu scolaire.

Paris : APLV.

Hornby, A.S. 1975. Guide to Patterns and Usage in English. $2^{\text {nd }}$ éd. Londres : Oxford University Press.

Larreya, P. et Rivière, C. 1991. Grammaire explicative de l'anglais. Paris : Longman.

McCawley, J.D. 1988. The Syntactic Phenomena of English, vol.1. Chicago \& Londres : University of Chicago Press, 207-261.

Quirk, R. \& S. Greenbaum. 1973. A University Grammar of English. Londres : Longman.

Rivière, C. 1988. Exercices commentés de grammaire anglaise. Paris : Ophrys.

Roggero, J. 1979. Grammaire anglaise. Paris : Nathan.

Schenker,V., P. Valentin et J. M.Zemb, J.M. 1969. Manuel du Germaniste. Paris : OCDL.

Strang, B. 1969. Modern English Structure. $2^{\text {nd }}$ éd. Londres : E. Arnold.

\section{ANNEXES}

\section{Annexe : tableaux des formes verbales en anglais}

Annexe 1. Actif (Phrase de départ : The children play in the garden.)

\begin{tabular}{|l|l|l|l|}
\hline MODAUX+ inf & HAVE + p.p. & BE + ing & VERBE \\
\hline $\begin{array}{l}\text { can/could } \\
\text { may/might } \\
\text { shall/should } \\
\text { will/would } \\
\text { must/ --- }\end{array}$ & have/has & $\begin{array}{l}\text { am/is/are } \\
\text { was/were } \\
\text { been }\end{array}$ & play \\
\hline & & ARE & playING \\
\hline & & & play \\
\hline
\end{tabular}




\begin{tabular}{|l|l|l|l|}
\hline & & WERE & playING \\
\hline & HAVE & & playED \\
\hline & HAVE & BEEN & playING \\
\hline & HAD & & playED \\
\hline WILL ${ }^{4}$ & HAD & BEEN & playING \\
\hline WILL & & & play \\
\hline WILL & HAVE & BE & playING \\
\hline WILL & HAVE & BEEN & playING \\
\hline WOULD & & & playED \\
\hline WOULD & & BE & playING \\
\hline WOULD & HAVE & & playED \\
\hline WOULD & HAVE & BEEN & playING \\
\hline
\end{tabular}

Annexe 2. Passif

\begin{tabular}{|l|l|l|l|l|}
\hline MODAUX +inf & HAVE + p.p. & BE + ing & BE +p.p. & Verbe \\
\hline & & & ARE & playED \\
\hline & & & WERE & playED \\
\hline & & are & BEing & playED \\
\hline & have & & BEing & playED \\
\hline & have & been & BEing & playED \\
\hline & had & & BEen & playED \\
\hline had & been & BEing & playED \\
\hline WILL & & & BE & playED \\
\hline WILL & & be & BEing & playED \\
\hline WILL & have & & BEen & playED \\
\hline
\end{tabular}




\begin{tabular}{|l|l|l|l|l|}
\hline WILL & have & been & BEing & playED \\
\hline WOULD & & & BE & playED \\
\hline WOULD & & be & BEing & playED \\
\hline WOULD & have & & BEen & playED \\
\hline WOULD & have & been & BEing & playED \\
\hline
\end{tabular}

\section{NOTES}

1. Étant donné les confusions fréquentes à ce niveau entre $B E+I N G$ et le passif, il a paru préférable de présenter seulement l'Actif dans un premier temps.

2. Lors de la discussion, Tony Lattes m'a suggéré d'introduire DO de la façon suivante : Aux -> F/

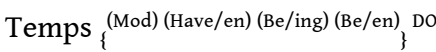

3. Dans les tableaux donnés en annexe, j'ai utilisé « Modaux + infinitif ». Ce terme peut prêter à confusion pour des étudiants habitués à «base verbale» ou «infinitif sans TO ». J'en ai tenu compte lors de la présentation.

4. Seuls WILL et WOULD sont ici pris comme exemples pour les modaux.

\section{RÉSUMÉS}

La formule de l'auxiliaire anglais d'après Chomsky (1957) est développée sous forme de tableau pour la rendre accessible à des étudiants d'IUT de tous niveaux. Ils peuvent ainsi reconstituer la structure verbale de n'importe quel verbe anglais. Cela permet aux «faux-débutants » en particulier de disposer d'une référence sûre et d'un moyen de contrôler leur production de structures verbales écrites. Par ailleurs, cela les aide également à mieux reconnaître et traduire les formes verbales complexes qu'ils rencontrent dans les textes techniques. Les avantages de cette présentation sont de donner confiance à ces étudiants en leur offrant une base de départ solide, et de susciter leur intérêt par une présentation scientifique et rigoureuse. Ceci est particulièrement vrai pour les étudiants d'électronique auxquels ce tableau a été présenté.

Chomsky's formula of the auxiliary in English (1957) is taken up and developed into a structural diagram so that it can be understood by IUT students of any level in English. They can thus reconstitute the structure of any English verb. This enables pre-intermediate students to have a reliable reference at hand and a means to check their production of written verbal structures. Furthermore, they find it easier to recognize and translate the complex verbal forms they come across in technical texts. The advantages of such a display are that a solid basis gives students more self-confidence and rouses their interest thanks to the scientific way in which it is presented. This was particularly true for students specializing in Electronics who were made familiar with the system. 
INDEX

Mots-clés : auxiliaire, faux-débutant, structure verbale

Keywords : auxiliary, pre-intermediate student, verb structure

\section{AUTEUR}

ALAIN DIANA

IUT 1, Grenoble 1. alain.diana@univ-paris-diderot.fr 